\title{
Abdominal drain amylase on the first day after pancreatectomy: a predictive factor for pancreatic fistula
}

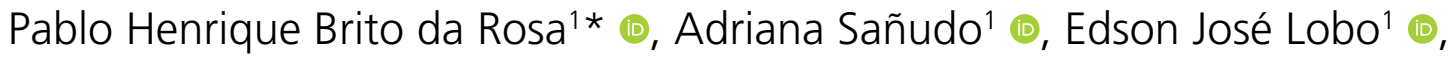 \\ Alberto Goldenberg ${ }^{1} \odot$, Gaspar de Jesus Lopes Filho ${ }^{\circledR}$, Franz Robert Apodaca Torrez ${ }^{1}$
}

\begin{abstract}
SUMMARY
OBJECTIVE: To analyze abdominal drain on the first postoperative day and evaluate its predictive nature for the diagnosis of Pancreatic Fistula exclusion, seeking to establish a cutoff point from which lower values demonstrate safety in excluding the possibility of this complication.

METHODS: From August 2017 to June 2020, data from 48 patients undergoing pancreatic resection were collected and analyzed from a prospective cohort. The patients were divided into two groups, one group consisting of patients who did not develop PF (Group A), and the other composed of patients who developed PF (Group B). The receiver operation characteristic curve was constructed, and cutoff points were evaluated by calculating sensitivity and specificity.

RESULTS: Group A brought 30 patients together (62.5\%) and Group B brought 18 patients together (37.5\%). The 444 U/L value was the most satisfactory cutoff point for the receiver operation characteristic curve ( $\mathrm{Cl} 0.690-0.941)$, with a sensitivity of $94.4 \%$ and a specificity of $60 \%$, thus being able to select 18 of 30 patients who did not succumb to PF.

CONCLUSIONS: Abdominal drain on the first postoperative day can be used as a predictive factor in the diagnosis of PF exclusion (Cl 0.690-0.941), with the value of $444 \mathrm{U} / \mathrm{L}$ being the best performance cutoff point.

KEYWORDS: Pancreatectomy. Pancreatic neoplasms. Amylases. Drainage. Pancreatic fistula.
\end{abstract}

\section{INTRODUCTION}

Pancreatic resection surgeries, such as pancreatoduodenectomy (PD) and distal pancreatectomy (DP) are the most common modalities in treating pancreatic neoplasms. Despite the improvement in the operative mortality rate for these surgeries in the last three decades, morbidity still remains high, with the pancreatic fistula (PF) being the main complication, as well as the most feared ${ }^{1-4}$.

To establish a definition for this complication, the International Pancreatic Fistula Study Group (ISGPF-2016) ${ }^{5}$ reviewed the literature and established that the content of the abdominal drain with an amylase dose greater than three times the serum limit, associated with a worsening of the patient's clinical condition from the third postoperative day defines the diagnosis of PF with clinical repercussion (PF-CR).

The considerable incidence of PF-CR has prompted other studies to find clinical and laboratory criteria predictive of which patients would evolve or would not with this complication. In this scenario the amylase from the drain on the first postoperative day $(\mathrm{AD} 1 \mathrm{PO})$ stands out, in which research has

'Universidade Federal de São Paulo, Department of Surgery - São Paulo (SP), Brazil.

*Corresponding author: bpablohenrique@gmail.com

Conflicts of interest: the authors declare there are no conflicts of interest. Funding: none.

Received on September 13, 2020. Accepted on November 08, 2020. 
been carried out to investigate whether this parameter can be used as a tool in the diagnostic exclusion of this complication, and thus asses the removal of the abdominal drain in the first postoperative $e^{6-12}$ days of. This practice, which has already been performed in some institutions, is associated with lower rates of intra-abdominal and pulmonary complications, length of hospital stay, and lower hospital costs ${ }^{13,14}$.

The data have shown that lower values in AD1PO are good indicators of exclusion in the diagnosis of PF, but the cutoff points vary widely between studies, thus lacking external validity $^{6-11,15,16}$. As such, this study analyzed the AD1PO in patients who underwent pancreatic resection surgery at Hospital São Paulo (São Paulo, Brazil).

\section{Objective}

To analyze the $\mathrm{AD} 1 \mathrm{PO}$ and evaluate its predictive nature in diagnosing exclusion of PF-CR, and to establish a cutoff point in which lower values (below) demonstrate safety to exclude diagnosis of this complication.

\section{METHODS}

The study was submitted and approved by the Research Ethics Committee with the Plataforma Brasil (CAAE) 94208718.7.0000.5505 and the Research Ethics Committee of UNIFESP 2.823.557.

A prospective cohort of patients with pancreatic neoplasms was instituted who underwent pancreatoduodenectomy, distal pancreatectomy, and pancreatic enucleation by the Group of Biliary Tract and Pancreas of the discipline of Surgical Gastroenterology of the Department of Surgery at UNIFESP at Hospital São Paulo from August 2017 to June 2020. Data were recorded on an Excel spreadsheet.

The measurement of amylase from the abdominal drain was performed on the first, third, and fifth postoperative days, with a diagnosis of PF-CR defined according to criteria of the ISGPF-20165. Epidemiological variables, postoperative evolution data, histopathological diagnosis, readmission, and mortality rates were also analyzed.

Patients were divided into two groups for statistical analysis of clinical and surgical variables: one group of patients developed PF-CR and the other group of patients did not deal with this complication. Statistical analysis was performed by Stata version 15.1, College Station, TX, USA. The categorical variables were measured in absolute and relative frequencies, quantitative variables in median and interquartile ranges (IQT), and mean and standard deviation as appropriate. To verify the association between groups in categorical variables, Fisher's exact test or the Chi-square test were used; for quantitative variables, the Student's T-test or the Mann-Whitney non-parametric test were used.

The Receiver Operating Characteristic Curve (ROC) was set up to assess the predictive component of AD1PO in the diagnosis of PF exclusion. From this curve, the cutoff points were evaluated by calculating sensitivity, specificity, positive predictive value (PPV), negative predictive value (NPV), accuracy, and likelihood ratio. The chosen cutoff point was the one with the highest sensitivity associated with the best specificity.

\section{RESULTS}

Within the period described, 48 patients underwent pancreatic resection surgery, and were divided into two groups, according to the presence or absence of PF-CR. Group A was comprised of 30 patients (62.5\%), 26 of whom did not develop PF (86.7\%), and four had a biochemical leak, without clinical repercussion (13.3\%). Group B was comprised of 18 patients (37.5\%), 11 of whom progressed to grade B PF (61.1\%), while seven progressed to grade C PF (38.9\%).

Patient characteristics are described in Table 1. Pancreatic adenocarcinoma was the main histological type of neoplasm in this sample. When analyzing groups $A$ and $B$, it was observed that pancreatic adenocarcinoma occurred with lower PF-CR rates $(22.2 \%)$, compared to other histological types (77.8\%).

For postoperative variables, with the exception of the number of deaths, other variables showed a statistically significant difference between groups $(\mathrm{p}<0.05)$. In group $\mathrm{B}, 16$ patients developed some postoperative complications (88.9\%); in group A, only six patients progressed poorly (20\%). Among the most frequent complications, abdominal collection was present in 15 patients in group B $(83.3 \%)$, and only one patient in group A $(3,3 \%)$. Days of hospitalization and days with the abdominal drain were higher in group B (30.5 and 33 days, respectively) vs. group A (11 and 10 days, respectively). The rate of readmission was also higher in group B, with $27.8 \%$ of patients being readmitted.

Table 1 shows the distribution of patients among the surgical modalities. Comparing surgical procedures and PF classification in patients who developed this complication, group $B$ saw a higher frequency of grade B PF in patients who underwent DP (87.5\%) compared to the group who underwent PD (44.4\%); however, this association did not present a statistically significant value, despite being borderline $(\mathrm{p}=0.06)$.

The AD1PO ROC curve, represented in Figure 1, showed an area under the curve equal to 0.815 , with a confidence interval (CI) of 0.690-0.941. The curve did not cross the null line and the $\mathrm{CI}$ was not lower than half (0.5), demonstrating that the $\mathrm{AD} 1 \mathrm{PO}$ presents good performance as a diagnostic test for 
Table 1. General Population Characteristics.

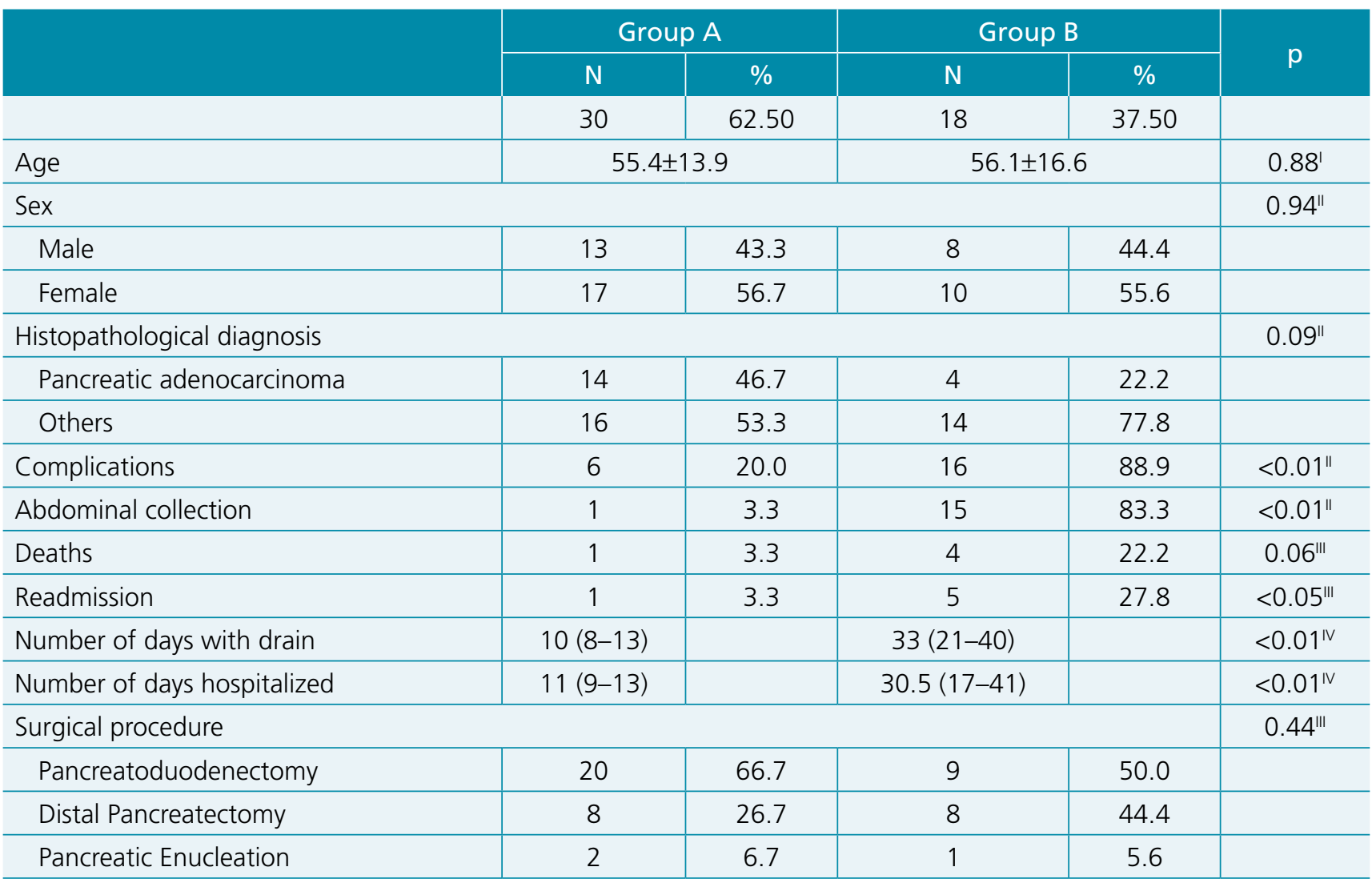

I: Student's T-test; II: $\chi^{2}$ test; III: Fisher's exact test; IV: Mann-Whitney test.

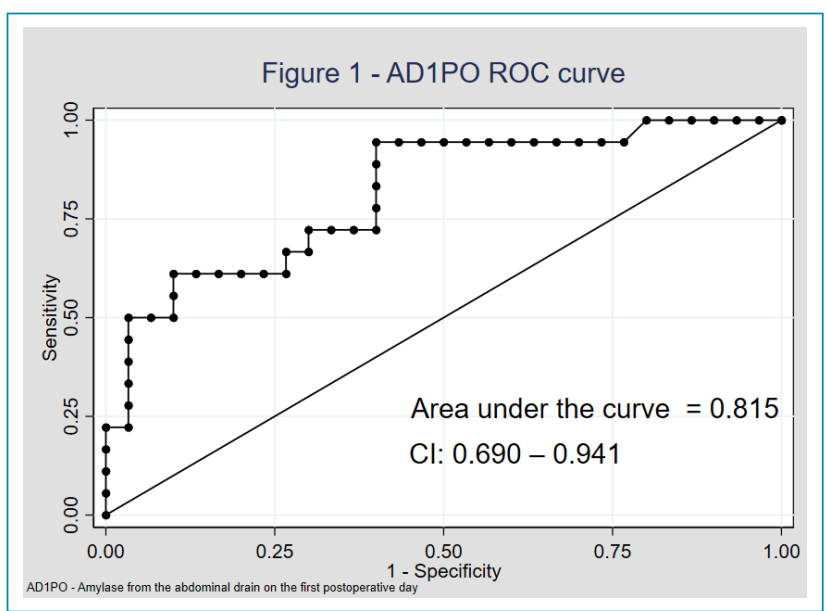

Figure 1. ROC curve of the amylase from the drain on the first postoperative day.

PF-CR. In Table 2, cutoff points were listed and evaluated to analyze exclusion capacity in the diagnosis of PF-CR. The cutoff point of $47 \mathrm{U} / \mathrm{L}$ showed higher sensitivity (100\%) and higher NPV (100\%) in this study; that is, none of the six patients below the cut had to deal with PF, being able to select $20 \%$ of patients in group A. The cutoff point of $444 \mathrm{U} / \mathrm{L}$ showed a sensitivity and NPV of about $94.4 \%$ and $94.7 \%$, respectively, with high clinical applicability, as 18 of 30 patients without PF were below the cutoff value, which addressed about $60 \%$ of patients in group A.

\section{DISCUSSION}

In pancreatic resection surgeries, placement of drains is a widely used measure, in contrast to other intra-abdominal surgeries (cholecystectomy, colectomy, hepatectomy, and splenectomy, for example $)^{17}$ for which this routine is not always indicated.

The Verona group ${ }^{13}$ chose 114 patients and divided them into two groups, which had early (3rd postoperative day) versus late removal of the drain (5th day or more). The result was that in the early group, we observed reduced rates of PF, intra-abdominal, and pulmonary complications, length of hospital stay, and lower hospital costs. However, the cutoff point of 5,000 U/L in the AD1PO includes patients with a high probability of developing a PF, as demonstrated in the study by Linneman et al. ${ }^{18}$ with 1,402 included patients, demonstrating a $7 \%$ incidence 
Tabela 2. Amylase from the drain on the first postoperative day cutoff points.

\begin{tabular}{l|c|c|c|c|c|c|c} 
Cutoff points & S (\%) & E (\%) & PPV (\%) & NPV (\%) & A (\%) & PLR & $\begin{array}{c}\text { Patients below } \\
\text { the cutoff with PF }\end{array}$ \\
\hline 47 & 100.0 & 20.0 & 42.8 & 100.0 & 50.0 & 1.25 & 0 \\
\hline 444 & 94.4 & 60.0 & 58.6 & 94.7 & 72.9 & 2.36 & 1 \\
\hline 634 & 77.8 & 60.00 & 53.8 & 81.8 & 66.7 & 1.94 & 4 \\
\hline
\end{tabular}

S: sensitivity; E: specificity; PPV: positivie predictive value; NPV: negative predictive value; A: accuracy; PLR: positive likelihood ratio

of $\mathrm{PF}$ in the group with $\mathrm{AD} 1 \mathrm{PO}<5,000 \mathrm{U} / \mathrm{L}$. Due to this fact, safer cutoff points have excluded the diagnosis of PF-CR.

Several studies evaluated $\mathrm{AD} 1 \mathrm{PO}$ as a predictive factor in the diagnosis of the exclusion of PF-CR ${ }^{6-12}$ to select patients at a low risk of developing this complication, and to remove abdominal drains in the first postoperative days ${ }^{13-14}$. Studies show favorable results and demonstrate that it can be used as a predictive factor in this situation; however, the cutoff points between studies have been quite variable.

The ROC curve of this study demonstrated that AD1PO can be used as a predictive factor for PF-CR. From this graph, cutoff points were analyzed, with the best performance value being $444 \mathrm{U} / \mathrm{L}$, selecting $60 \%$ of 30 patients who did not have PF-CR; however, this cutoff point missed one patient who was forced to deal with PF-CR, with sensitivity and NPV not reaching $100 \%$, as shown in Table 2. It should be noted that this patient, even with a low AD1PO, maintained the hypothesis of PF: other clinical criteria, including the patient's general condition, level of consciousness, and the issue of the drained secretion were also altered, factors which are considered in assessing the prognosis of $\mathrm{PF}$ associated with an abdominal infectious focus.

The cutoff point of this study was lower than that by Fong et al. ${ }^{11}$ In their initial cohort of 126 patients who underwent pancreatoduodenectomy, a cutoff point of $612 \mathrm{U} / \mathrm{L}$ was estimated, with a sensitivity of $93 \%$ and a specificity of $79 \%$. After this analysis, a validation cohort was assessed with 369 patients, divided into two groups, the first composed of patients with AD1PO below $600 \mathrm{U} / \mathrm{L}$, and the other exceeding or equaling that value. In the first group of $62.1 \%$ patients, only two developed into PF-CR, demonstrating that this cutoff point was satisfactory for the sample.

There are some systematic reviews evaluating the studies of $\mathrm{AD} 1 \mathrm{PO}$ and their predictive character in the diagnosis of $\mathrm{PF}$ and PF-CR ${ }^{15,18}$. All came to the conclusion that, despite evidence showing that $\mathrm{AD} 1 \mathrm{PO}$ has a high predictive capacity for PF-CR, there are several cutoff points that can vary between institutions; that is, the external validity of these values is the main limitation of the studies. In the meta-analysis by Giglio et al. ${ }^{15}$, they analyzed cutoff intervals to guarantee safety and rule out a diagnosis of PF-CR, which would select a number of patients with a low risk of developing the complication. Performing this analysis, the value of $350 \mathrm{U} / \mathrm{L}$ showed a sensitivity of $91 \%$ (CI 76-97\%) and a specificity of $84 \%$ (CI 59-95\%), a value close to that of $444 \mathrm{U} / \mathrm{L}$.

An important limitation of this study was the joint analysis of three surgical modalities: PD, DP, and pancreatic enucleation (EN). Due to the low number of PD and DP, there was not a high incidence of $\mathrm{PF}$ in relation to the others with any statistical significance, nor an analysis of AD1PO between the two groups was performed due to the reduced number of patients.

The decision for permanence or removal of the drain varies according to protocols of each hospital; however, data such as the behavior of the amylase drain for several days, appearance of the drained effluent content, and the clinical condition of the patient are criteria when deciding to keep or remove the drain. Using AD1PO as the only decisive criterion to decide on its removal can lead to errors in a minority of cases, as observed in this study. Thus, combining clinical criteria with a low dosage of $\mathrm{AD} 1 \mathrm{PO}$ can be a viable alternative in maintaining or removing the abdominal drain in the first postoperative days.

\section{CONCLUSION}

$\mathrm{AD} 1 \mathrm{PO}$ can be used as a predictive factor in the diagnostic exclusion of PF-CR, because its ROC curve did not exceed the nullity line $(\mathrm{CI} 0.690-0.941)$, with the value of $444 \mathrm{U} / \mathrm{L}$ being the best performance point, since it was able to detect about $60 \%$ of patients who did not develop PF, in addition to presenting high sensitivity and NPV.

\section{AUTHORS" CONTRIBUTIONS}

PHBR: Conceptualization, Data Curation, Writing Original Draft. AS: Data Curation Formal Analysis. EJL: Conceptualization, Methodology, Writing - Review \& Editing. AG: Conceptualization, Methodology, Writing - Review \& Editing. GJLF: Conceptualization, Methodology, Writing Review \& Editing. FAT: Conceptualization, Methodology, Writing - Review \& Editing. 


\section{REFERENCES}

1. Greenblatt DY, Kelly KJ, Rajamanickam V, Wan Y, Hanson T, Rettammel $R$, et al. Preoperative factors predict perioperative morbidity and mortality after pancreaticoduodenectomy. Ann Surg Oncol. 2011;18(8):2126-35. https://doi.org/10.1245/ s10434-011-1594-6

2. Newhook TE, LaPar DJ, Lindberg JM, Bauer TW, Adams RB, Zaydfudim VM. Morbidity and mortality of pancreaticoduodenectomy for benign and premalignant pancreatic neoplasms. J Gastrointest Surg. 2015;19(6):1072-7. https://doi.org/10.1007/s11605-015-2799-y

3. Gouma DJ, van Geenen RC, van Gulik TM, Haan RJ, Wit LT, Busch OR, et al. Rates of complications and death after pancreaticoduodenectomy: risk factors and the impact of hospital volume. Ann Surg. 2000;232(6):786-95. https://doi. org/10.1097/00000658-200012000-00007

4. Grobmyer SR, Pieracci FM, Allen PJ, Brennan MF, Jaques DP. Defining morbidity after pancreaticoduodenectomy: use of a prospective complication grading system. J Am Coll Surg. 2007;204(3):356-64. https://doi.org/10.1016/j. jamcollsurg.2006.11.017

5. Bassi C, Marchegiani G, Dervenis C, Sarr M, Abu Hilal M, Adham M, et al. The 2016 update of the International Study Group (ISGPS) definition and grading of postoperative pancreatic fistula: 11 Years After. Surgery. 2017;161(3):584-91. https:// doi.org/10.1016/j.surg.2016.11.014

6. Amico EC, Azevedo IM, Fernandes MVL, Reis MA, João SA. Amilase do dreno no primeiro dia de pós-operatório de operaçao de Whipple: Qual valor é melhor preditor para a retirada precoce do dreno? ABCD, Arq Bras Cir Dig. 2018;31(1):e1345. https://doi.org/10.1590/0102-672020180001e1345

7. Bertens KA, Crown A, Clanton J, Alemi F, Alseidi AA, Biehl $T$, et al. What is a better predictor of clinically relevant postoperative pancreatic fistula (CR-POPF) following pancreaticoduodenectomy (PD): postoperative day one drain amylase (POD1DA) or the fistula risk score (FRS)? HPB (Oxford). 2017;19(1):75-81. https://doi.org/10.1016/j.hpb.2016.10.001

8. Molinari E, Bassi C, Salvia R, Butturini G, Crippa Stefano, Talamini $\mathrm{G}$, et al. Amylase value in drains after pancreatic resection as predictive factor of postoperative pancreatic fistula: results of a prospective study in 137 patients. Ann Surg. 2007;246(2):281-7. https://doi.org/10.1097/SLA.0b013e3180caa42f

9. Sutcliffe RP, Battula N, Haque A, Ali A, Srinivasan P, Atkinson $S W$, et al. Utility of drain fluid amylase measurement on the first postoperative day after pancreaticoduodenectomy. World J Surg. 2012;36(4):879-83. https://doi.org/10.1007/s00268012-1460-0
10. Maggino L, Malleo G, Bassi C, Allegrini V, Beane JD, Beckman RM, et al. Identification of an Optimal Cut-off for Drain Fluid Amylase on Postoperative Day 1 for Predicting Clinically Relevant Fistula After Distal Pancreatectomy: A Multi-institutional Analysis and External Validation. Ann Surg. 2019;269(2):33743. https://doi.org/10.1097/SLA.0000000000002532

11. Ven Fong Z, Correa-Gallego C, Ferrone CR, Veillette GR, Warshaw AL, Lillemoe KD, et al. Early Drain Removal--The Middle Ground Between the Drain Versus No Drain Debate in Patients Undergoing Pancreaticoduodenectomy: A Prospective Validation Study. Ann Surg. 2015:262(2):378-83. https://doi. org/10.1097/SLA.0000000000001038

12. Tanioka T, Kojima K, Saito T, Kanemoto E, Okuno K, Gokita $\mathrm{K}$, et al. Intraoperative Body Fluid Amylase as a Novel Indicator of Postgastrectomy Pancreatic Fistula. World J Surg. 2019:43(8):2061-8. https://doi.org/10.1007/s00268-019-04961-9

13. Bassi C, Molinari E, Malleo G, Crippa S, Butturini G, Salvia $R$, et al. Early versus late drain removal after standard pancreatic resections: results of a prospective randomized trial. Ann Surg. 2010;252(2):207-14. https://doi.org/10.1097/ SLA.0b013e3181e61e88

14. McMillan MT, Malleo G, Bassi C, Allegrini V, Casetti L, Drebin JA, et al. Multicenter, Prospective Trial of Selective Drain Management for Pancreatoduodenectomy Using Risk Stratification. Ann Surg. 2017;265(6):1209-18. https://doi. org/10.1097/SLA.0000000000001832

15. Giglio MC, Spalding DR, Giakoustidis A, Le Bian AZ, Jiao LR, Habib NA, et al. Meta-analysis of drain amylase content on postoperative day 1 as a predictor of pancreatic fistula following pancreatic resection. Br J Surg. 2016;103(4):328-36. https:// doi.org/10.1002/bjs. 10090

16. Liu $Y, L i Y$, Wang $L$, Peng CJ. Predictive value of drain pancreatic amylase concentration for postoperative pancreatic fistula on postoperative day 1 after pancreatic resection: An updated meta-analysis. Medicine (Baltimore). 2018;97(38):e12487. https://doi.org/10.1097/MD.0000000000012487

17. Petrowsky H, Demartines N, Rousson V, Clavien PA. Evidencebased value of prophylactic drainage in gastrointestinal surgery: a systematic review and meta-analyses. Ann Surg. 2004;240(6):1074-85. https://doi.org/10.1097/01. sla.0000146149.17411.c5

18. Linnemann RJA, Patijn GA, van Rijssen LB, Besselink MG, Mungroop $\mathrm{TH}$, Hingh $\mathrm{IH}$, et al. The role of abdominal drainage in pancreatic resection - A multicenter validation study for early drain removal. Pancreatology. 2019;19(6):888-96. https:// doi.org/10.1016/j.pan.2019.07.04119 